\title{
Refinement of Propagation Techniques of Aonla (Emblica officinalis Gaertn.) in North Western Himalayan Region
}

\author{
Sanjeev K. Banyal* and Ajay Kumar Banyal
}

Dept. of Fruit Science, Dr. Y.S. Parmar University of Horticulture and Forestry College of Horticulture and Forestry, Neri, Hamirpur, H.P. (177 001), India

\section{Corresponding Author}

Sanjeev K. Banyal

e-mail: skbanyal@gmail.com

\author{
Article History \\ Article ID: AR1938 \\ Received in $10^{\text {th }}$ December, 2018 \\ Received in revised form $20^{\text {th }}$ February, 2019 \\ Accepted in final form $28^{\text {th }}$ February, 2019
}

\begin{abstract}
The study was undertaken to refine nursery production technology on aonla in order to maximize nursery output. Healthy seedlings of aonla cultivars NA-10 and NA-7 were transplanted at three spacing and were applied three fertilizer doses. In Another experiment the seedlings were transplanted on five $\left(15^{\text {th }}\right.$ and $30^{\text {th }}$ June, $15^{\text {th }}$ and $30^{\text {th }}$ July and $15^{\text {th }}$ August) dates to find out the optimum time for transplanting of aonla rootstock. It was also observed during the study that the seedling survival and plant growth in spacing $S_{1}\left(30 \times 30 \mathrm{~cm}^{2}\right)$ were low as compared to other spacing, which resulted in poor budding success and ultimately lesser number of saleable plant. In spacing $\mathrm{S}_{3}(30 \times 10$ $\mathrm{cm}^{2}$ ) the initial plant population was very low, although, the seedling survival, growth parameters and budding success were highest in combination having $\mathrm{S}_{3}$ spacing, final production of saleable plants was lower than spacing $\mathrm{S}_{2}\left(30 \times 20 \mathrm{~cm}^{2}\right)$. There was a definite effect of budding time on budding success. The seedlings budded in July had better bud taken as compared to those budded in March. However, the seedlings transplanted on $15^{\text {th }}$ July observed to have the maximum budding success both during March and July. The rate of successful budding increased steadily up to third transplanting date ( $\left.15^{\text {th }} \mathrm{July}\right)$ and declined thereafter. Aonla cultivar NA-10 had better budding success $(52.87 \%)$ in the plants budded in March whereas the effect was non-significant during July.
\end{abstract}

Keywords: Aonla, Emblica officinalis, budding, nursery, fertilization

\section{Introduction}

Aonla (Emblica officinalis Gaertn.) is one of the most important minor fruits and a crop of commercial significance. It belongs to the family Euphorbiaceae. It is quite hardy, prolific bearer and much remunerative even without much care. Its importance lies in its high richness of vitamin $\mathrm{C}$ next only to Barbados cherry which is not grown in India. Aonla is one of the hardiest fruit trees highly suitable for cultivation in arid and semi-arid regions of the country. It is of high commercial significance because of its unique medicinal and industrial values. Thus it can become a promising source of remuneration as well as good health for the families of poor farmers.

Aonla is believed to have been originated in tropical, south eastern Asia, particularly in central and southern India. It is also reported to be the native of India, Sri Lanka, Malaysia and China where it grows well and is found growing in wild form. In India, its commercial cultivation is done in Utter Pradesh. Pratapgarh, Varanasi, Azamgarh, Sultanpur, etc. are the famous aonla growing districts of Uttar Pradesh. Aonla contains 500-1500 mg of ascorbic acid per $100 \mathrm{~g}$ of pulp (Chauhan et al., 2005) which is twenty times more than oranges. Being highly rich in ascorbic acid content, it is used to treat a number of maladies. Aonla is one of the most suitable fruit crop for plantation in the wastelands due to its high productivity (116 mt ha-1, NHB, 2014), drought tolerance and lesser incidence to pests and diseases.

Shortage of quality planting material of established cultivars is a major bottleneck in area expansion of aonla in Himachal Pradesh. Majority of plantation is still done through seedlings which bear small fruits of inferior quality. Propagation of aonla through chip budding is most commercialized method but rate of success is low due to various reasons. The time of transplanting has significant effect on attachment of buddable seedlings. There is a lack of specific information on exact time of seed sowing, transplanting of seedlings, spacing fertilizers doses in the nursery and budding time. Thus the studies were initiated to standardize fertilizer doses, time of transplanting and spacing for aonla under subtropical conditions of Himachal Pradesh.

\section{Materials and Methods}

An experiment was conducted to work out optimum spacing and fertilizer level for rapid nursery production of aonla on the experimental farm of Institute of Biotechnology and 
Environmental Science, Neri during 2012-13 and and 201314. The experimental site is located at an altitude of $620 \mathrm{~m}$ above mean sea level with latitude $31^{\circ} 68^{\prime} \mathrm{N}$ and longitude $76^{\circ}$ $52^{\prime} \mathrm{E}$. The mean minimum and maximum temperature ranges between $12.4{ }^{\circ} \mathrm{C}$ to $31.3{ }^{\circ} \mathrm{C}$ and average humidity remains around $60.91 \%$ (Figure 1 and 2). The soil in the experimental area was clay loam with a $\mathrm{pH}$ of 6.6 and had $0.38 \%$ organic matter content. Soil $\mathrm{N}$ and $\mathrm{P}$ were low and $\mathrm{K}$ was medium. Healthy seedlings of aonla cultivars NA-10 and NA-7 were transplanted at three spacing and were applied three fertilizer

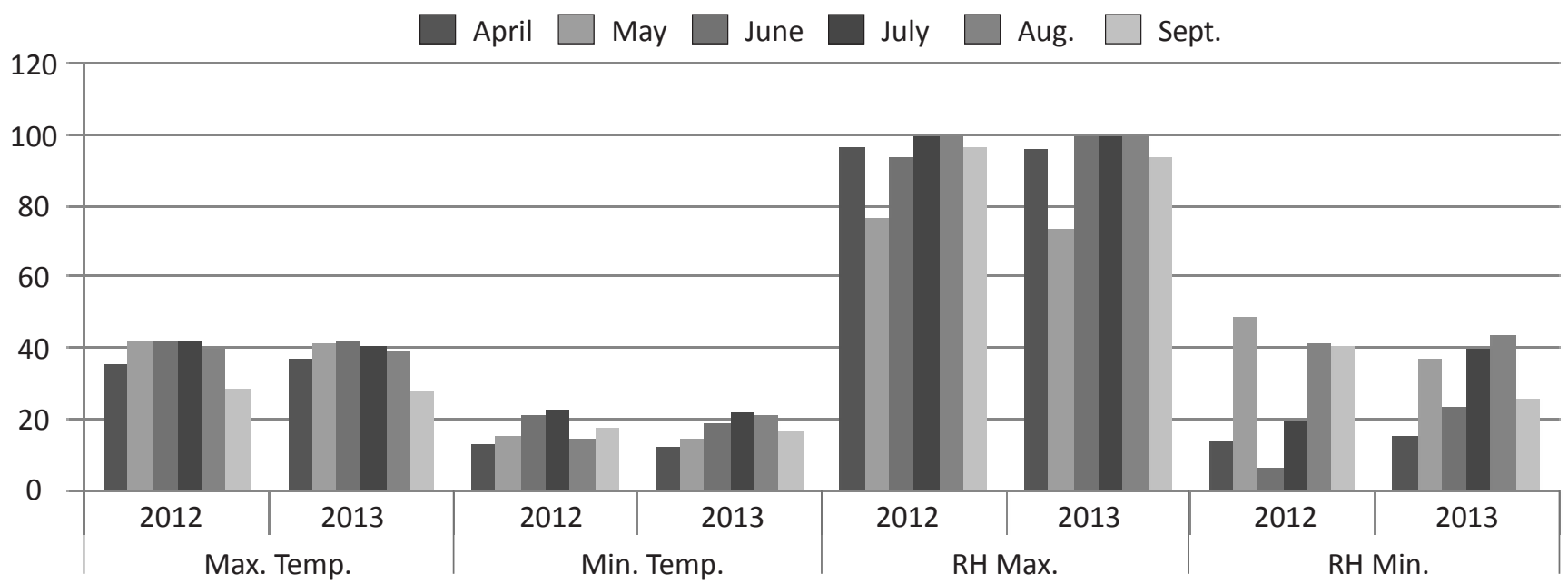

Figure 1: Meteorological data of experimental site

Rainfall $2012 \square$ Rainfall 2013

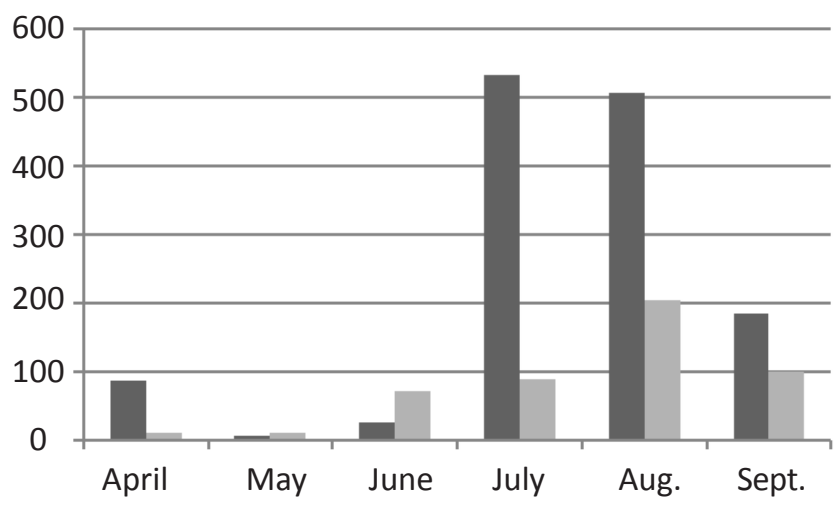

Figure 2: Rainfall data of experimental site

doses (Table 1).

The resulting 18 treatment combinations were laid out in randomized block design with three replications. In Another experiment the seedlings were transplanted on five $\left(15^{\text {th }}\right.$ and $30^{\text {th }}$ June, $15^{\text {th }}$ and $30^{\text {th }}$ July and $15^{\text {th }}$ August) dates to find out the optimum time for transplanting of aonla rootstock. These seedlings were budded during the impending March and July. The observations were recorded on seedling survival, length and girth of seedlings, buddable seedlings, budding success and saleable plants produced per unit area. The data obtained during two years of study was pooled as per the method suggested by Gomez and Gomez (1984).

\section{Results and Discussion}

\subsection{Effect of cultivars, spacing and fertilizers}

\subsubsection{Seedling survival, height and girth}

Various treatment combinations had a significant effect

\begin{tabular}{|c|c|c|c|}
\hline Varieties & Spacing & Fertilizers & $\begin{array}{l}\text { Treatment } \\
\text { combinations }\end{array}$ \\
\hline $\mathrm{V} 1=\mathrm{NA}-7$ & $\mathrm{~S}_{1}=30 \times 10 \mathrm{~cm}^{2}$ & $\begin{array}{l}\mathrm{F}_{1}=10 \mathrm{Kg} \mathrm{FYM+} \\
25 \mathrm{~g} \mathrm{~N}+16 \mathrm{~g} \\
\mathrm{P}_{2} \mathrm{O}_{5}+60 \mathrm{~g} \mathrm{~K}_{2} \mathrm{O} \\
\mathrm{m}^{-2} \text { bed area }\end{array}$ & $\begin{array}{l}V_{1} S_{1} F_{1}, V_{1} S_{1} F_{1}, \\
V_{1} S_{1} F_{3} \\
V_{1} S_{2} F_{1}, V_{1} S_{2} F_{2} \\
V_{1} S_{2} F_{3}\end{array}$ \\
\hline \multirow[t]{2}{*}{$V_{2}=N A-10$} & $\mathrm{~S}_{2}=30 \times 20 \mathrm{~cm}^{2}$ & $\begin{array}{l}\mathrm{F}_{2}=5 \mathrm{~kg} \mathrm{FYM+} \\
12.5 \mathrm{~g} \mathrm{~N}+8 \mathrm{~g} \\
\mathrm{P}_{2} \mathrm{O}_{5}+30 \mathrm{~g} \mathrm{~K}_{2} \mathrm{O} \\
\mathrm{m}^{-2} \text { bed area }= \\
\left(1 / 2 \text { of } \mathrm{F}_{1}\right)\end{array}$ & $\begin{array}{l}V_{1} S_{3} F_{1}, V_{1} S_{3} F_{2}, \\
V_{1} S_{3} F_{3} \\
V_{2} S_{1} F_{1}, V_{2} S_{1} F_{2}, \\
V_{2} S_{1} F_{2} \\
V_{2} S_{2} F_{1}, V_{2} S_{2} F_{2}\end{array}$ \\
\hline & $\mathrm{S}_{3}=30 \times 30 \mathrm{~cm}^{2}$ & $\begin{array}{l}\mathrm{F}_{3}=20 \mathrm{~kg} \mathrm{FYM+} \\
50 \mathrm{~g} \mathrm{~N}+32 \mathrm{~g} \\
\mathrm{P}_{2} \mathrm{O}_{5}+120 \mathrm{~g} \\
\mathrm{~K}_{2} \mathrm{O} \mathrm{m}^{-2} \text { bed } \\
\text { area }=\text { (Twice } \\
\text { of } \mathrm{F}_{1} \text { ) }\end{array}$ & $\begin{array}{l}V 2 S 2 F 3 \\
V_{2} S_{3} F_{1}, V_{2} S_{3} F_{2} \\
V_{2} S_{3} F_{3}\end{array}$ \\
\hline
\end{tabular}

on seedling survival, height and girth, budding success and production of saleable plants (Table 2). Plant survival was found to be maximum (51.50\%) in treatment combination $\mathrm{V}_{1} \mathrm{~S}_{3} \mathrm{~F}_{3}$ and was statistically at par with $\mathrm{V}_{2} \mathrm{~S}_{3} \mathrm{~F}_{3}(50.99 \%), \mathrm{V}_{1} \mathrm{~S}_{3} \mathrm{~F}_{2}$ $(50.07 \%)$ and five other combinations. Similar results were obtained for seedling height and girth which were found to have maximum values (56.75 and $0.93 \mathrm{~cm}$, respectively) in treatment combination $\mathrm{V}_{1} \mathrm{~S}_{3} \mathrm{~F}_{3}$. Percentage of buddable seedlings ranged between $54.59 \%\left(V_{1} S_{3} F_{3}\right)$ and $42.02 \%\left(V_{1} S_{1} F_{1}\right)$. Eleven treatment combinations were statistically at par with lowest value for this trait. 
Table 2: Effect of cultivars, spacing and fertilizers on nursery production in aonla (Emblica officinalis Gaertn.) (Pooled data for two years)

\begin{tabular}{lcccccc}
\hline Treatment & Seedling survival (\%) & $\begin{array}{c}\text { Height } \\
(\mathrm{cm})\end{array}$ & $\begin{array}{c}\text { Girth } \\
(\mathrm{cm})\end{array}$ & $\begin{array}{c}\text { Buddable } \\
\text { seedlings (\%) }\end{array}$ & $\begin{array}{c}\text { Budding success } \\
(\%)\end{array}$ & $\begin{array}{c}\text { No. of saleable } \\
\text { plants m }\end{array}$ \\
\hline $\mathrm{V}_{1} \mathrm{~S}_{1} \mathrm{~F}_{1}$ & $44.14(41.64)$ & 36.11 & 0.56 & $44.81(42.02)$ & $53.03(46.73)$ & 5.14 \\
$\mathrm{~V}_{1} \mathrm{~S}_{1} \mathrm{~F}_{2}$ & $46.25(42.85)$ & 39.25 & 0.59 & $46.55(43.02)$ & $53.21(46.84)$ & 5.87 \\
$\mathrm{~V}_{1} \mathrm{~S}_{1} \mathrm{~F}_{3}$ & $47.29(43.45)$ & 43.15 & 0.61 & $48.23(43.98)$ & $55.11(47.93)$ & 5.99 \\
$\mathrm{~V}_{1} \mathrm{~S}_{2} \mathrm{~F}_{1}$ & $46.34(42.90)$ & 38.13 & 0.66 & $52.96(46.70)$ & $59.77(50.63)$ & 7.11 \\
$\mathrm{~V}_{1} \mathrm{~S}_{2} \mathrm{~F}_{2}$ & $49.45(44.68)$ & 41.19 & 0.68 & $54.27(47.45)$ & $63.15(52.62)$ & 7.78 \\
$\mathrm{~V}_{1} \mathrm{~S}_{2} \mathrm{~F}_{3}$ & $51.88(46.08)$ & 43.80 & 0.71 & $57.35(49.29)$ & $66.53(54.65)$ & 7.93 \\
$\mathrm{~V}_{1} \mathrm{~S}_{3} \mathrm{~F}_{1}$ & $56.17(48.55)$ & 47.19 & 0.77 & $59.23(50.32)$ & $68.12(55.62)$ & 4.09 \\
$\mathrm{~V}_{1} \mathrm{~S}_{3} \mathrm{~F}_{2}$ & $58.80(50.07)$ & 53.90 & 0.84 & $61.25(51.50)$ & $70.63(57.18)$ & 4.76 \\
$\mathrm{~V}_{1} \mathrm{~S}_{3} \mathrm{~F}_{3}$ & $61.25(51.50)$ & 56.75 & 0.93 & $66.43(54.59)$ & $74.27(59.52)$ & 4.90 \\
$\mathrm{~V}_{2} \mathrm{~S}_{1} \mathrm{~F}_{1}$ & $41.45(40.08)$ & 34.94 & 0.49 & $40.63(39.60)$ & $49.29(44.59)$ & 5.03 \\
$\mathrm{~V}_{2} \mathrm{~S}_{1} \mathrm{~F}_{2}$ & $48.02(43.87)$ & 37.16 & 0.52 & $43.25(41.12)$ & $52.00(46.14)$ & 5.70 \\
$\mathrm{~V}_{2} \mathrm{~S}_{1} \mathrm{~F}_{2}$ & $51.63(45.93)$ & 40.62 & 0.57 & $47.11(43.34)$ & $53.69(47.11)$ & 5.96 \\
$\mathrm{~V}_{2} \mathrm{~S}_{2} \mathrm{~F}_{1}$ & $53.11(46.78)$ & 44.37 & 0.62 & $49.95(44.97)$ & $56.17(48.54)$ & 6.87 \\
$\mathrm{~V}_{2} \mathrm{~S}_{2} \mathrm{~F}_{2}$ & $53.98(47.28)$ & 46.11 & 0.67 & $51.63(45.93)$ & $59.43(50.43)$ & 7.51 \\
$\mathrm{~V}_{2} \mathrm{~S}_{2} \mathrm{~F}_{3}$ & $55.35(48.07)$ & 49.53 & 0.73 & $54.22(47.42)$ & $60.72(51.19)$ & 7.66 \\
$\mathrm{~V}_{2} \mathrm{~S}_{3} \mathrm{~F}_{1}$ & $57.15(49.11)$ & 54.47 & 0.81 & $57.60(49.37)$ & $63.22(52.66)$ & 4.11 \\
$\mathrm{~V}_{2} \mathrm{~S}_{3} \mathrm{~F}_{2}$ & $58.00(49.61)$ & 55.63 & 0.86 & $59.83(50.67)$ & $67.15(55.03)$ & 4.69 \\
$\mathrm{~V}_{2} \mathrm{~S}_{3} \mathrm{~F}_{3}$ & $60.37(50.99)$ & 57.91 & 0.90 & $61.15(51.44)$ & $68.23(55.69)$ & 4.94 \\
$\mathrm{Mean}_{2}$ & $52.26(46.30)$ & 45.56 & 0.69 & $53.13(46.81)$ & $60.76(51.28)$ & 5.89 \\
$\mathrm{LSD}_{0.05}$ & 6.26 & 13.76 & 0.23 & 9.65 & 6.98 & 1.74 \\
\hline
\end{tabular}

\subsubsection{Budding success}

The highest budding success (59.52\%) was also recorded in the combination $\mathrm{V}_{1} \mathrm{~S}_{3} \mathrm{~F}_{3}$ which was at par with $\mathrm{V}_{1} \mathrm{~S}_{3} \mathrm{~F}_{2}(57.18 \%)$, $\mathrm{V}_{2} \mathrm{~S}_{2} \mathrm{~F}_{3}(55.69 \%), \mathrm{V}_{1} \mathrm{~S}_{3} \mathrm{~F}_{1}(55.62 \%)$ and $\mathrm{V}_{2} \mathrm{~S}_{3} \mathrm{~F}_{2}(55.03 \%)$. In general it was noticed that treatment combination involving low planting density $\left(30 \times 30 \mathrm{~cm}^{2}\right)$ and higher fertilizer application rate $\left(F_{3}\right)$ produced significantly higher values of all parameters under study. It was observed that treatment combination with spacing of $30 \times 30 \mathrm{~cm}^{2}\left(\mathrm{~S}_{3}\right)$ recorded highest seedling survival rate which may be attributed to enhanced availability of moisture and nutrients at low planting density. Saroj and Singh (2010) conducted an experiment on refinement of aonla propagation through budding under hot and arid ecosystem. The treatment comprised of nine filling mixtures and four sizes of polybags. The results revealed that polybags of $25 \times 15$ $\mathrm{cm}^{2}$ size either filled with field sandy soil+pond soil+compost $(1: 1: 1)$ or field sandy soil+FYM (1:2) gave more than $80 \%$ success after patch budding in the month of June, which induced more than $45 \mathrm{~cm}$ of budling growth in the month of July. These treatment combinations had also given better seed germination, percentage rootstock survival, height and girth of rootstocks, longer and fibrous root system. Negi et al. (2010) carried out the study to standardize the most suitable method of propagation among patch budding, shield budding, chip budding, forkert budding and wedge grafting. The results revealed that among the 5 methods of orchard establishment, transplanting of one month old seedlings/rootstocks raised in polybags recorded the highest bud sprouting $(69.33 \%)$ as well as bud/graft survival (79.18\%). Among the 5 methods of propagation, patch budding had the highest percentage of success $(80.33 \%)$, closely followed by the chip method (77.67\%). Chip budding resulted in the highest bud/graft survival of $80.71 \%$ followed by patch budding $(78.89 \%)$. Chip budding also took a minimum of 11.27 and 15.27 days for initiation and $50 \%$ completion of bud sprouting.

\subsubsection{Production of saleable plants}

Highest (7.93) number of saleable plants $\mathrm{m}^{2}$ was recorded in treatment combination $\mathrm{V}_{1} \mathrm{~S}_{2} \mathrm{~F}_{3}$ which was at par with $\mathrm{V}_{2} \mathrm{~S}_{2} \mathrm{~F}_{3}$ (7.66), $V_{1} S_{2} F_{2}(7.43)$ and $V_{2} S_{2} F_{2}(7.36)$. It was seen that spacing $\mathrm{S}_{2}\left(30 \times 20 \mathrm{~cm}^{2}\right)$ was the common among the combinations producing highest number of saleable plants. It was also observed during the study that the seedling survival and plant growth in spacing $S_{1}\left(30 \times 30 \mathrm{~cm}^{2}\right)$ were low as compared to other spacing, which resulted in poor budding success and ultimately lesser number of saleable plants whereas, in 
spacing $\mathrm{S}_{3}\left(30 \times 10 \mathrm{~cm}^{2}\right)$ the initial plant population was very low, although, the seedling survival, growth parameters and budding success were highest in combination having $\mathrm{S}_{3}$ spacing, final production of saleable plants was lower than spacing $\mathrm{S}_{2}\left(30 \times 20 \mathrm{~cm}^{2}\right)$. Similar results were also obtained by Banyal and Sharma (2011) who recorded highest number of saleable plants $/ \mathrm{m}^{2}$ (11.64) in mango when nursery was transplanted at a spacing of $30 \times 20 \mathrm{~cm}^{2}$. Choudhary et al., 2016 conducted an experiment to investigate the success and growth of softwood grafts of different aonla (Emblica officinalis gaertn.) varieties at nursery stage. Considering the overall performance of grafts of different aonla varieties studied, the result revealed that the grafts of varieties NA-7 and Banarasi have performed better for most of the traits under this study.

\subsection{Effect of time of transplanting}

Date of transplanting was found to have significant effect on survival, growth, budding success and proportion of saleable plants (Table 3). Percent survival increased when the seedlings were transplanted from $15^{\text {th }}$ June $(28.87 \%)$ to $15^{\text {th }}$ of July (55.39\%). Thereafter it steadily decreased upto $15^{\text {th }}$ August (33.99\%). There was no significant difference among varieties. The treatmentxvariety interaction was significant for plant survival. The treatmentxvariety interaction was significant for plant survival. Proportion of buddable seedlings was also recorded to be maximum (51.92\%) when the seedlings were transplanted on $15^{\text {th }}$ July $\left(\mathrm{T}_{3}\right)$ and thereafter a steady decrease was observed upto $15^{\text {th }}$ August (34.57\%) which recorded the lowest percentage of buddable seedlings. Among

\begin{tabular}{|c|c|c|c|c|c|c|c|c|c|c|c|c|}
\hline \multirow{3}{*}{$\begin{array}{l}\text { Trans - } \\
\text { planting } \\
\text { dates }\end{array}$} & \multirow{2}{*}{\multicolumn{3}{|c|}{ Plant survival (\%) }} & \multirow{2}{*}{\multicolumn{3}{|c|}{ Buddable seedlings (\%) }} & \multicolumn{6}{|c|}{ Budding success (\%) } \\
\hline & & & & & & & \multicolumn{3}{|c|}{ Budded in March } & \multicolumn{3}{|c|}{ Budded in July } \\
\hline & $\begin{array}{c}\mathrm{V}_{1} \\
(\mathrm{NA}-10)\end{array}$ & $\begin{array}{c}\mathrm{V}_{2} \\
(\mathrm{NA}-7)\end{array}$ & Mean & $\begin{array}{c}V_{1} \\
(N A-10)\end{array}$ & $\begin{array}{c}\mathrm{V}_{2} \\
(\mathrm{NA}-7)\end{array}$ & Mean & $\begin{array}{c}\mathrm{V}_{1} \\
(\mathrm{NA}-10)\end{array}$ & $\begin{array}{c}\mathrm{V}_{2} \\
(\mathrm{NA}-7)\end{array}$ & Mean & $\begin{array}{c}\mathrm{V}_{1} \\
(\mathrm{NA}-10)\end{array}$ & $\begin{array}{c}\mathrm{V}_{2} \\
(\mathrm{NA}-7)\end{array}$ & Mean \\
\hline $\begin{array}{l}\mathrm{T}_{1}\left(15^{\text {th }}\right. \\
\text { June })\end{array}$ & $\begin{array}{c}30.40 \\
(33.46)\end{array}$ & $\begin{array}{c}27.34 \\
(31.52)\end{array}$ & $\begin{array}{c}28.87 \\
(32.49)\end{array}$ & $\begin{array}{c}51.67 \\
(45.95)\end{array}$ & $\begin{array}{c}41.09 \\
(39.86)\end{array}$ & $\begin{array}{c}46.38 \\
(42.91)\end{array}$ & $\begin{array}{c}54.33 \\
(47.48)\end{array}$ & $\begin{array}{c}51.66 \\
(45.95)\end{array}$ & $\begin{array}{c}52.99 \\
(46.71)\end{array}$ & $\begin{array}{c}60.33 \\
(50.96)\end{array}$ & $\begin{array}{c}64.00 \\
(53.13)\end{array}$ & $\begin{array}{c}62.16 \\
(52.04)\end{array}$ \\
\hline $\begin{array}{l}\mathrm{T}_{2}\left(30^{\text {th }}\right. \\
\text { June })\end{array}$ & $\begin{array}{c}33.15 \\
(35.15)\end{array}$ & $\begin{array}{c}31.56 \\
(34.18)\end{array}$ & $\begin{array}{c}32.35 \\
(34.66)\end{array}$ & $\begin{array}{c}57.78 \\
(49.47)\end{array}$ & $\begin{array}{c}54.33 \\
(47.48)\end{array}$ & $\begin{array}{c}56.05 \\
(48.48)\end{array}$ & $\begin{array}{c}65.66 \\
(54.12)\end{array}$ & $\begin{array}{c}57.33 \\
(49.21)\end{array}$ & $\begin{array}{c}61.49 \\
(51.67)\end{array}$ & $\begin{array}{c}67.00 \\
(54.94)\end{array}$ & $\begin{array}{c}70.00 \\
(56.79)\end{array}$ & $\begin{array}{c}68.5 \\
(55.86)\end{array}$ \\
\hline $\begin{array}{l}\mathrm{T}_{3}\left(15^{\text {th }}\right. \\
\text { July) }\end{array}$ & $\begin{array}{c}57.35 \\
(49.22)\end{array}$ & $\begin{array}{c}54.23 \\
(47.42)\end{array}$ & $\begin{array}{c}55.79 \\
(48.32)\end{array}$ & $\begin{array}{c}64.29 \\
(53.30)\end{array}$ & $\begin{array}{c}59.62 \\
(50.54)\end{array}$ & $\begin{array}{c}61.95 \\
(51.92)\end{array}$ & $\begin{array}{c}71.33 \\
(57.62)\end{array}$ & $\begin{array}{c}68.00 \\
(55.55)\end{array}$ & $\begin{array}{c}69.66 \\
(56.59)\end{array}$ & $\begin{array}{c}79.66 \\
(63.19)\end{array}$ & $\begin{array}{c}81.33 \\
(64.40)\end{array}$ & $\begin{array}{c}80.49 \\
(63.79)\end{array}$ \\
\hline $\begin{array}{l}\mathrm{T}_{4}\left(30^{\text {th }}\right. \\
\text { July) }\end{array}$ & $\begin{array}{c}49.97 \\
(44.98)\end{array}$ & $\begin{array}{c}47.25 \\
(43.42)\end{array}$ & $\begin{array}{c}48.61 \\
(44.20)\end{array}$ & $\begin{array}{c}55.44 \\
(48.12)\end{array}$ & $\begin{array}{c}48.35 \\
(44.05)\end{array}$ & $\begin{array}{c}51.89 \\
(46.09)\end{array}$ & $\begin{array}{c}68.00 \\
(55.55)\end{array}$ & $\begin{array}{c}61.66 \\
(51.74)\end{array}$ & $\begin{array}{c}64.83 \\
(53.64)\end{array}$ & $\begin{array}{c}71.66 \\
(57.83)\end{array}$ & $\begin{array}{c}67.66 \\
(55.34)\end{array}$ & $\begin{array}{c}69.66 \\
(56.59)\end{array}$ \\
\hline $\begin{array}{l}\mathrm{T}_{5}\left(15^{\text {th }}\right. \\
\text { August) }\end{array}$ & $\begin{array}{c}37.22 \\
(37.59)\end{array}$ & $\begin{array}{c}30.76 \\
(33.68)\end{array}$ & $\begin{array}{c}33.99 \\
(35.64)\end{array}$ & $\begin{array}{c}32.35 \\
(34.66)\end{array}$ & $\begin{array}{c}36.80 \\
(37.34)\end{array}$ & $\begin{array}{c}34.57 \\
(36.00)\end{array}$ & $\begin{array}{c}58.00 \\
(49.60)\end{array}$ & $\begin{array}{c}47.33 \\
(43.47)\end{array}$ & $\begin{array}{c}52.66 \\
(46.53)\end{array}$ & $\begin{array}{c}64.33 \\
(53.32)\end{array}$ & $\begin{array}{c}63.33 \\
(52.73)\end{array}$ & $\begin{array}{c}63.83 \\
(53.03)\end{array}$ \\
\hline Mean & $\begin{array}{c}41.62 \\
(40.08)\end{array}$ & $\begin{array}{c}38.23 \\
(38.04)\end{array}$ & & $\begin{array}{c}52.31 \\
(46.30)\end{array}$ & $\begin{array}{c}48.04 \\
(43.86)\end{array}$ & & $\begin{array}{c}63.46 \\
(52.87)\end{array}$ & $\begin{array}{c}57.19 \\
(49.18)\end{array}$ & & $\begin{array}{c}68.60 \\
(56.05)\end{array}$ & $\begin{array}{c}69.26 \\
(56.48)\end{array}$ & \\
\hline $\operatorname{LSD}_{0.05}$ & $\begin{array}{l}\text { Treatmer } \\
\text { Variety ( } \\
\text { TxV }\end{array}$ & $\begin{array}{l}\text { t }(T) \\
\text { ) }\end{array}$ & $\begin{array}{c}5.33 \\
\text { NS } \\
8.27\end{array}$ & & $\begin{array}{c}7.35 \\
2.21 \\
17.45\end{array}$ & & & $\begin{array}{c}6.58 \\
3.55 \\
15.36\end{array}$ & & & $\begin{array}{c}5.87 \\
\text { NS } \\
12.08\end{array}$ & \\
\hline
\end{tabular}

Table 3: Continue...

\begin{tabular}{|c|c|c|c|c|c|c|}
\hline \multirow[t]{3}{*}{ Transplanting dates } & \multicolumn{6}{|c|}{ Saleable plants (\%) } \\
\hline & \multicolumn{3}{|c|}{ Budded in March } & \multicolumn{3}{|c|}{ Budded in July } \\
\hline & $V_{1}(N A-10)$ & $V_{2}$ (NA-7) & Mean & $V_{1}$ (NA-10) & $V_{2}$ (NA-7) & Mean \\
\hline$T_{1}\left(15^{\text {th }}\right.$ June $)$ & $64.45(53.40)$ & $59.25(50.33)$ & $61.85(51.86)$ & $52.13(46.22)$ & $47.16(43.37)$ & 49.64 (44.79) \\
\hline $\mathrm{T}_{2}\left(30^{\text {th }}\right.$ June $)$ & $72.58(58.42)$ & $67.22(55.07)$ & $69.90(65.74)$ & $61.25(51.50)$ & $55.80(48.33)$ & 58.52 (49.91) \\
\hline $\mathrm{T}_{3}\left(15^{\text {th }}\right.$ July $)$ & $81.96(64.86)$ & $78.20(62.16)$ & $80.08(63.51)$ & $70.28(56.96)$ & $64.33(53.32)$ & $67.30(55.14)$ \\
\hline $\mathrm{T}_{4}\left(30^{\text {th }}\right.$ July $)$ & $43.15(41.06)$ & $44.11(41.61)$ & $43.63(41.34)$ & $36.25(37.02)$ & $29.44(32.86)$ & $32.84(34.94)$ \\
\hline$T_{5}\left(15^{\text {th }}\right.$ August $)$ & 11.25 (19.59) & $9.35(17.80)$ & $10.30(18.70)$ & $13.43(21.49)$ & $10.40(18.81)$ & $11.91(20.15)$ \\
\hline Mean & $54.67(47.47)$ & $51.62(45.39)$ & & $46.66(42.64)$ & $41.42(39.34)$ & \\
\hline \multirow{3}{*}{$\mathrm{LSD}_{0.05}$} & Treatment $(\mathrm{T})$ & \multicolumn{2}{|c|}{18.14} & \multicolumn{3}{|c|}{16.04} \\
\hline & Variety (V) & \multicolumn{2}{|c|}{2.04} & \multicolumn{3}{|c|}{3.26} \\
\hline & $\mathrm{T} \times \mathrm{V}$ & \multicolumn{2}{|c|}{24.18} & \multicolumn{3}{|c|}{23.45} \\
\hline
\end{tabular}


varieties, NA-10 had significantly higher number of buddable seedlings (46.30) as compared to NA-7. Similar results were also reported by Sharma et al., 2000. They observed highest percentage of seedling survival on $30^{\text {th }}$ July, maximum vegetative growth and budding success on $15^{\text {th }}$ July in Aonla, under similar climatic conditions. In a study conducted by Nanawar (2006), NA-10 variety recorded significantly maximum length of sprouted shoot at $90(13.69 \mathrm{~cm})$ and 120 $(17.60 \mathrm{~cm})$ days. The combination of NA-10 variety budded/ grafted on $15^{\text {th }}$ August showed significantly maximum final survival percentage $58.57 \%$ of budded/grafted plants after 120 days. There was a definite effect of budding time on budding success. The seedlings budded in July had better bud taken as compared to those budded in March. However the seedlings transplanted on $15^{\text {th }}$ July observed to have the maximum budding success both during March and July. The rate of successful budding increased steadily upto third transplanting date ( $15^{\text {th }}$ July) and declined thereafter. Aonla cultivar NA-10 had better budding success $(52.87 \%)$ in the plants budded in March whereas the effect was non-significant during July. Kaur and Kaur (2018) carried out similar experiment to study the standardization of propagation techniques in jamun. The treatment comprises of ten planting times commencing from May to February and three methods of propagation i.e. T- budding, patch budding and cleft grafting. The results of the study indicated that among planting time $1-15^{\text {th }}$ August proved to be the most suitable in improving the root, shoot and leaf parameters. Maximum number of leaves/plant, stem thickness was recorded in cleft grafting propagation technique in jamun.

\section{Conclusion}

Maximum number of saleable plants (63.51\%) was recorded in the plants transplanted on $15^{\text {th }}$ June and budded in February. Among all the transplanting dates, the plants budded in March had the higher percentage of saleable plants as compared to those budded in July. The possible reason for this could be the more growing period available to the plants budded in March. The percentage of saleable plants decreased sharply as they were transplanted after $15^{\text {th }}$ July. Both the cultivars were statistically similar with respect to production of number of saleable plants.

\section{References}

Chauhan, O.P. Srivastava, S., Pandey, P., Rao, G.K., 2005. A study on the development of aonla blended sauce. Beverage and Food world 32, 31-33.

Choudhary, S.M., Kadam, A.S., Ramniwas and Chavan, D.L., 2016. Success and growth of softwood grafts of different aonla (Emblica officinalis gaertn.) Varieties at nursery stage. The Bioscan, an international quarterly journal of life sciences 11(4), 2533-2536.

Gomez, K.W., Gomez A.A., 1984. Statistical procedures for agricultural research. John Wiley \& Sons.

Kaur, H., Kaur, A., 2018. Standardization of Propagation Techniques in Jamun. International Journal of Current Microbiology and Applied Sciences 7(10), 2143-2151.

Nanaware, M.A., 2006. studies to standardize propagation techniques in aonla (Emblica officinalis Gaertn. M.Sc. thesis. Mahatma Phule Krishi Vidyapeeth, Rahuri-413 722. Dist. Ahmednagar, Maharastra, India

Negi, R.S., Baghel, B.S., Gupta, A.K., 2010. Standardization of method of orchard establishment and propagation in Aonla (Emblica officinalis Gaertn.) for rehabilitation of degraded pasture/grazing lands. Progressive Horticulture 42(2), 173-178.

NHB, 2014. Handbook of Horticultural statistics. http://nhb. gov.in/default.aspx.

Pathak, R.K., 2003. Status report on genetic resources of Indian Gooseberry-Aonla (Emblica officinalis Gaertn.) in South and Southeast Asia. IPGRI Office for South Asia National Agriculture Science Centre (NASC) DPS Marg, Pusa Campus, New Delhi 110 012, India.

Saroj, P.L., Singh, U.V., 2010. Refinement of aonla propagation through budding under hot arid ecosystem. Indian Journal of Horticulture 67, 90-95.

Sharma, A.K., Sharma, K., Badyala, S.D., 2000. Effect of transplanting time of rootstock and budding technique on rapid propagation of aonla (Emblica officinalis Gaertn.). Indian Journal of Soil Conservation 28(3), 235-237.

Sharma, S., Kumar and Banyal, Kumar, S., 2002. Nursery output maximization in mango under low hill conditions of Himachal Pradesh. Journal of Horticultural Sciences 6(1), 56-58.

Shrivastava, A.L., Singh, H.K., Singh, I.S., 2002. Studies on propagation of aonla (Emblica officinalis). Haryana Journal of Horticultural Sciences 31(3-4)156-158. 